\title{
Hypereosinophiliesyndrome
}

\section{Hypereosinophilic Syndromes}

Autoren

Institute
C. Houriet ${ }^{1}$, H.-U. Simon ${ }^{2}$, D. Simon ${ }^{1}$

${ }^{1}$ Universitätsklinik für Dermatologie, Inselspital Bern, Schweiz

${ }^{2}$ Institut für Pharmakologie, Universität Bern, Schweiz
Bibliografie

Dol http://dx.doi.org/

10.1055/s-0034-1365078

Online-Publikation: 26.2.2014

Akt Dermatol 2014; 40: 127-132

(c) Georg Thieme Verlag KG

Stuttgart · New York

ISSN 0340-2541

Korrespondenzadresse

Prof. Dr. Dagmar Simon

Universitätsklinik für

Dermatologie

Inselspital

CH-3010 Bern

Schweiz

dagmar.simon@insel.ch

\section{Zusammenfassung \\ $\nabla$}

In den vergangenen Jahren wurden zahlreiche neue Erkenntnisse zur Biologie eosinophiler Granulozyten, ihrer Rolle für die Gesundheit und bei Krankheiten gewonnen. Differenzierte Kenntnisse zur Pathogenese, neue diagnostische Methoden und Therapeutika haben das Verständnis zur Hypereosinophilie und damit assoziierten Erkrankungen und Syndromen fundamental geändert. In dieser Übersichtsarbeit diskutieren wir die gegenwärtige Klassifikation der Hypereosinophiliesyndrome und neue Therapieansätze.

\section{Einleitung}

Über vierzig Jahren basierte die Diagnose des Hypereosinophiliesyndroms auf den drei von Chusid et al. [1] definierten Kriterien: 1) Bluteosinophilie $>1,5 \times 10^{9} / 1$, gemessen in einem zeitlichen Abstand von mehr als sechs Monaten, 2) fehlende Hinweise auf eine allergische, parasitäre Erkrankung oder andere bekannte Ursachen einer Eosinophilie, 3) Zeichen einer Eosinophilen-assoziierten Endorganschädigung [2]. Seitdem haben sich unser Wissen über die Pathogenese eosinophiler Erkrankungen sowie die diagnostischen und therapeutischen Möglichkeiten extrem erweitert, sodass sich diese ursprüngliche Definition mit der Zeit als problematisch und limitierend im klinischen Alltag erwies. Zum Beispiel erscheint es nicht angemessen, sechs Monate auf die Diagnose und damit Therapieeinleitung zu warten. Zudem wurde die Liste von Erkrankungen, die eine Hypereosinophilie bedingen, erweitert und spezifiziert. Mit der alten Definition wurde der Tatsache, dass aktivierte Eosinophile im Gewebe frühzeitig eine Gewebedestruktion ohne begleitende Bluteosinophilie von $\geq 1,5 \times 10^{9} / 1$ verursachen können, zu wenig Beachtung geschenkt [2]. Je besser die verschiedenen Ursachen der Hypereosinophilie erklärt werden konnten, desto klarer wurde, dass es sich nicht um ein Syndrom sondern um mehrere Hypereosinophiliesyndrome handelt.

In dieser Übersicht wollen wir die gegenwärtige Diskussion zur Definition und Klassifikation der Hypereosinophiliesyndrome aufgreifen und einige praktische Hinweise für die Diagnostik, die nicht selten von den Dermatologen ausgeht, und therapeutische Ansatzpunkte herausarbeiten.

\section{Die Biologie der Eosinophilen \\ $\nabla$}

Eosinophile Granulozyten entstehen im Knochenmark aus CD34+-hämatopoetischen Vorläuferzellen und werden anschließend ins periphere Blut abgegeben, wo sie bei Gesunden 1-5\% der gesamten Leukozytenzahl oder in absoluten Zahlen maximal $0,5 \times 10^{9} / 1$ ausmachen $[3,4]$. Vor allem Interleukin (IL)-5 wie auch IL-3 und Granulocyte Macrophage Colony-Stimulating Factor (GM-CSF) stellen die wichtigsten Stimulationsfaktoren für die Produktion, Differenzierung und Überleben der Eosinophilen dar [3-5]. Sie werden von aktivierten T-Zellen, Mastzellen, Stromazellen und von den Eosinophilen selbst produziert und bewirken nicht nur das normale Wachstum, sondern sind zudem für die Migration, Adhäsion, Aktivierung und Zytokinproduktion von Eosinophilen notwendig [3-5]. Die Mobilisierung der Eosinophilen vom Knochenmark ins periphere Blut wird vor allem durch IL-5 und Eotaxin reguliert [3-5].

Physiologischerweise kommen Eosinophile in verschiedenen lymphatischen und hamätopoetischen Geweben wie Knochenmark, Thymus, Milz und Lymphknoten aber auch in der Mukosa des Magens, Dünn- und Dickdarmes und im Uterus vor. Andere gesunde Gewebe enthalten keine Eosinophilen [3]. Prinzipiell kann im Rahmen einer eosinophilen Erkrankung jedes Organ von Eosinophilen infiltriert werden $[3,4]$. 
Eosinophile produzieren eine Vielzahl biologisch aktiver Mediatoren wie basische Granulaproteine (Eosinophilic Cationic Protein [ECP], Major Basic Protein [MBP], Eosinophil-Derived Neurotoxin [EDN] und Eosinophilenperoxidase [EPO]), außerdem Prostaglandine, Leukotriene, Thromboxan A und verschiedene Zytokine [6]. Kürzlich wurde gezeigt, dass Eosinophile extrazelluläre Netze generieren, die aus mitochondraler DNA in Assoziation mit Granulaproteinen bestehen und in der Lage sind, Bakterien zu töten $[7,8]$. Diese sogenannten Eosinophil Extracellular Traps (EET) lassen sich im Gewebe infektiöser und nicht-infektiöser wie allergischer Erkrankungen [7,9] unter anderem bei vielen verschiedenen eosinophilen Hauterkrankungen nachweisen [10-12]. Außerdem konnten wir entsprechend der Ätiologie verschiedene Zytokinexpressionsmuster der Eosinophilen nachweisen [13].

Eine Eosinophilie kann transient, episodisch oder anhaltend auftreten und infolge der Aktivierung und Ausschüttung ihrer oben genannten Mediatoren eine Störung von Organfunktionen bedingen und bis hin zu potenziell irreversiblen Organschädigungen führen $[14,15]$. Der genaue Mechanismus der Endorganschädigung durch eine persistierende Hypereosinophilie ist nicht vollständig geklärt. Mittels Ausschüttung toxischer Granulaproteine können die Eosinophilen direkt Mikroorganismen und Zellen töten sowie lokal eine Entzündungsreaktion initiieren. Zusätzlich trägt die Produktion proinflammatorischer Zytokine dazu bei, dass andere Entzündungszellen rekrutiert und aktiviert werden. Über eine Schädigung und Aktivierung von Endothelzellen und Thrombozyten sowie Freisetzung prothrombotischer Faktoren können Eosinophile direkt zur Thrombenbildung und Fibrose beitragen [15]. Die endokardiale Fibrose sowie eine erhöhte Thromboseneigung gelten als lebensbedrohliche Komplikationen bei Hypereosinophilie.

Basierend auf diesen Erkenntnissen werden heute den Eosinophilen verschiedene Rollen zugeschrieben: die Abwehr von Mikroorganismen, eine immunregulatorische Funktion, Gewebezerstörung sowie der Gewebeumbau wie Fibrosierung/Remodelling [5].

\section{Hypereosinophilie im Blut und Gewebe}

$\nabla$

Gemäß den gängigen Kriterien wird die Eosinophilie in eine milde $\left(0,5-1,5 \times 10^{9} / 1\right)$, mittelschwere $\left(>1,5 \times 10^{9} / 1\right)$ und schwere $\left(>5 \times 10^{9} / 1\right)$ Form unterteilt.

2011 kam der Vorschlag einer Konsensusgruppe, den Begriff der Hypereosinophilie bei persistierender Eosinophilie von $>1,5 \times$ $10^{9} / 1$ und/oder bei Vorliegen einer erheblichen Gewebeeosinophilie zu verwenden [16]. Diese sollte zweimal in Abständen von mindestens 4 Wochen vorliegen, im Gegensatz zur ursprünglichen Definition von Chusid et al. [1], die einen Abstand von 6 Monaten verlangte. Als Ausnahme gilt eine durch die Eosinophilie verursachte Endorganschädigung, die eine umgehende Therapie erfordert [16].

Von Hypereosinophiliesyndromen wird dann gesprochen, wenn die Kriterien einer Hypereosinophilie erfüllt sind und eine Gewebeschädigung infolge der Hypereosinophilie vorliegt $[1,16]$.

Von einer erheblichen Gewebehypereosinophilie spricht man, wenn 1. im Knochenmark die relative Zahl der Eosinophilen über 20\% der Gesamtzellzahl ausmacht und/oder 2. gemäß Pathologen eine übermäßige Infiltration durch Eosinophile im Vergleich zu normalem Gewebe verzeichnet wird und/oder 3. mittels spezifischer Färbungen vermehrte extrazelluläre Ablagerungen eosinophiler Granulaproteine nachgewiesen werden können [16]. Eine Gewebehypereosinophilie kann mit oder ohne Bluteosinophilie einhergehen [16].

Verschiedene Organsysteme können infolge einer Hypereosinophilie eine Schädigung aufweisen, meistens sind jedoch Herz, Lungen, Haut (Erytheme, Ödem, Ulzeration, Ekzem), Milz, Gastrointestinaltrakt und Zentralnervensystem betroffen [15-17]. Zudem können konstitutionelle Symptome wie Müdigkeit, rezidivierende Fieberschübe und Myalgien auftreten [16].

\section{Neue Vorschläge zur Klassifikation der Hypereosinophilie \\ $\nabla$}

Eine wichtige Überlegung zur Einteilung der Hypereosinophilie zielt auf deren Pathogenese ab. Neben der idiopathischen ( $\mathrm{HE}_{\mathrm{US}}$ ) und familiären Hypereosinophile $\left(\mathrm{HE}_{\mathrm{FA}}\right)$ kristallisieren sich zwei Hauptmechanismen heraus, die eine Eosinophilie bewirken können: eine primäre, neoplastische Expansion von Eosinophilen im Rahmen einer klonalen myeloiden, eosinophilen oder Stammzellerkrankung $\left(\mathrm{HE}_{\mathrm{N}}\right)$ und eine sekundäre, reaktive Form mit nicht-klonaler Eosinophilenvermehrung infolge nicht- oder paraneoplastischer Erkrankungen $\left(\mathrm{HE}_{\mathrm{R}}\right)(\bullet$ Abb. 1) $[16,18]$. Es wird also unterschieden, ob die Ursache der Hypereosinophilie in (intrinsische Form) oder außerhalb (extrinsische Form) der Zelllinie der Eosinophilen liegt [18]. Zu den intrinsischen eosinophilen Erkrankungen würde man hämatologische Erkrankungen zählen, welche durch Mutationen in multi- oder pluripotenten hämatopoetischen Stammzellen bedingt sind [18]. Im Gegensatz dazu umfassen extrinsische Erkrankungen jene Hypereosinophilien, die durch eine vermehrte Produktion von eosinophilopoetischen Zytokinen, hier insbesondere IL-5, IL-3 und GM-CSF, entstehen [18]. Bei letzterer, auch reaktiv genannter Hypereosinophilie, handelt es sich um eine zytokinvermittelte Steigerung der Produktion, Aktivierung und/oder Lebensdauer der Eosinophilen [18]. Diese Zytokine können von Lymphozyten, meist aktivierten klonalen oder nicht-klonalen T-Zellen, als auch Tumorzellen produziert werden [19-21].

\section{Zytokingetriggerte reaktive Hypereosinophilie}

Eine reaktive Hypereosinophilie wird am häufigsten bei entzündlichen Erkrankungen, beispielsweise bei Infektionen mit Helminthen, aber auch bei einer fortgeschrittenen HIV-1-Infektion beobachtet. Allergische oder toxische Arzneimittelreaktionen und atopische Erkrankungen wie Rhinokonjunktivitis allergica, Asthma bronchiale, atopisches Ekzem sowie eosinophile Ösophagitis (EoE) führen durch die Aktivierung von T-Zellen und die anschließende Produktion von T-Helfer-2-Zytokinen, vor allem IL-5, zu einer Eosinophilie [15, 16, 18]. Seltene Ursachen der Hypereosinophilie stellen die chronische Graft-Versus-Host-Erkrankung (GVHD), verschiedene Autoimmunerkrankungen (z.B. Dermatomyositis, Systemischer Lupus erythematodes, Sjögren-Syndrom, primäre billiäre Zirrhose, bullöses Pemphigoid) und die allergische bronchopulmonale Aspergillose dar $[16,18]$.

Auch im Rahmen einer Neoplasie lassen sich reaktive Hypereosinophilien beobachten. Beispiele sind hämatologische Neoplasien, wie das Hodgkin-Lymphom, T- oder B-Zelllymphome einschließlich kutaner Lymphome wie die Mycosis fungoides und die BLymphoblastische Leukämie [15]. Desweiteren sind paraneoplastische Eosinophilien bei soliden Tumoren wie Adenokarzinome der Lunge, der Cervix oder des Gastrointestinaltraktes beschrie- 


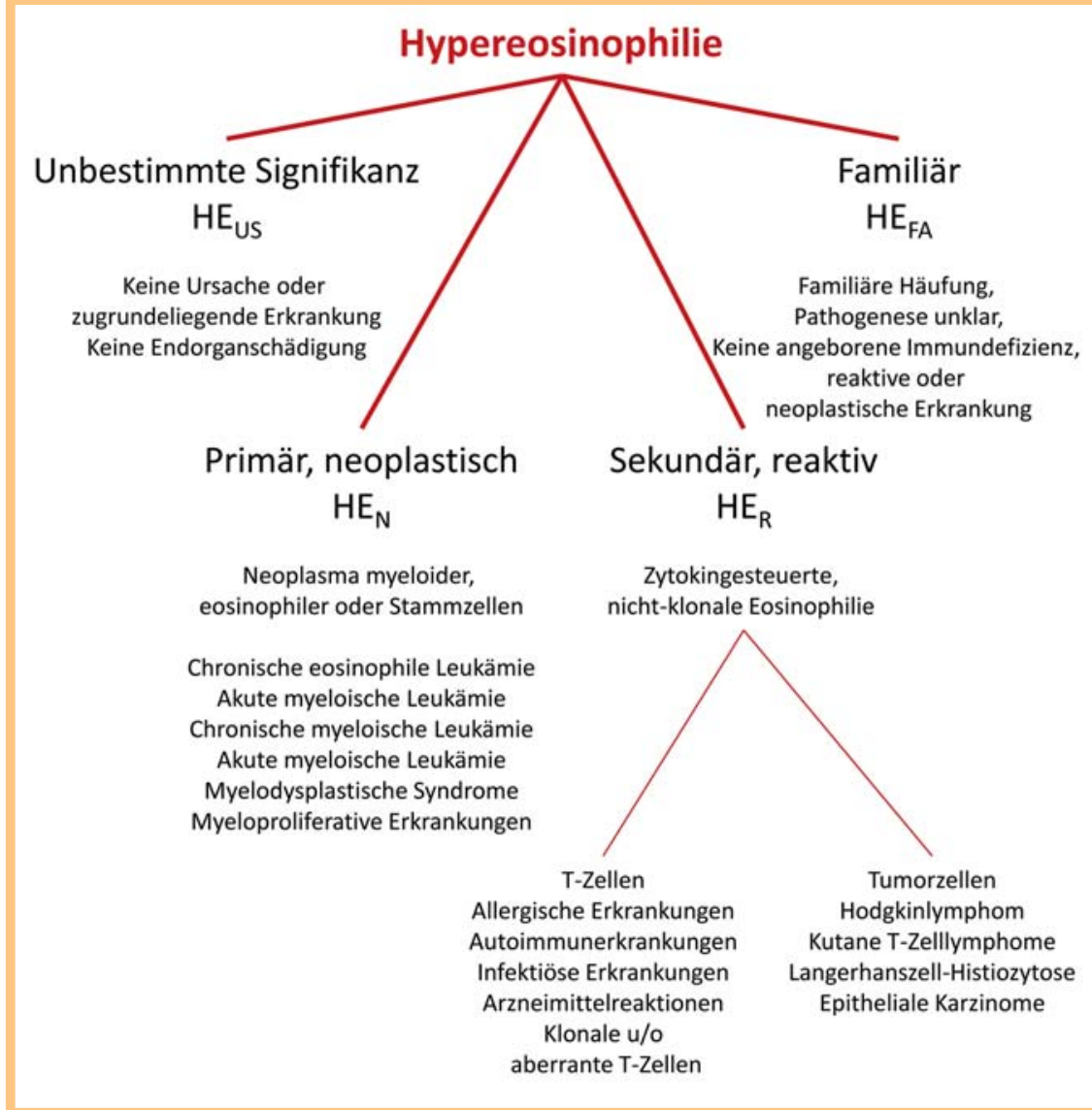

Abb.1 Klassifikation der Hypereosinophilie [16].

ben $[15,19,22]$. In den meisten Fällen kommt es zu einer vermehrten Produktion von eosinophilopoetischen Zytokinen, wie IL-3 und IL-5, die eine sekundäre polyklonale Blut- und/oder Gewebseosinophilie verursachen $[2,15,16]$. Außerdem wurden klonale (und nicht-klonale) Vermehrungen von T-Zellen mit atypischem Phänotyp als Ursache der Hypereosinophilie berichtet, die als lympozytäre Variante zusammengefasst werden $[16,20]$. Ein Übergang in ein T-Zelllymphom wurde, wenn auch in seltenen Fällen, beobachtet [21].

\section{Intrinsische Hypereosinophilie \\ $\nabla$}

Eosinophilie ist ein häufig auftretendes Zeichen verschiedener hämatologischer Malignome (neoplastische Hypereosinophilie). Die primäre Ursache der Eosinophilie liegt in einer Mutation hämatopoetischer pluri- oder multipotenter Vorläuferzellen und nicht wie bei der reaktiven Hypereosinophilie in einer vermehrten Produktion von eosinophilopoetischen Zytokinen [2,14].

Neoplasien der myeloiden Zellreihen, die häufig mit einer Hypereosinophilie einhergehen, beinhalten die chronische myeloische Leukämie, die chronische eosinophile Leukämie, andere myeloproliferative Neoplasien, akute und chronische Leukämien, fortgeschrittene systemische Mastzellerkrankungen und seltene Formen des myelodysplastischen Syndroms [14,16]. Die Klassifikation der myeloproliferativen Erkrankungen mit einer Hypereosinophilie erfolgt nach den Richtlinien der WHO [14, $16,23,24]$. Die chronische eosinophile Leukämie (CEL) stellt eine Sonderform der chronischen myeloischen Leukämie dar, bei der die Eosinophilen den hauptsächlichen Klon ausmachen. Es wurden verschiedene Entitäten beschrieben, in ca. 10 - 20\% aller Fälle kann eine Fusion des Gens für PDGFRA (platelet derived growth factor alpha) mit FIP1L1 gefunden werden, was eine erhöhte Aktivität dieser Tyrosinkinase zur Folge hat [15]. Die endomyokardiale Fibrose als gefürchtete Komplikation einer Hypereosinophilie wird häufig bei CEL mit Nachweis des Fusionsproteins FIP1L1-PDGFRA, nicht aber bei anderen myeloiden Neoplasien beobachtet [14-16]. Die Entdeckung der PDGFRA-assoziierten CEL war bahnbrechend, da nun eine Reihe früher als idiopathisch bezeichneter Hypereosinophiliesyndrome klassifiziert und einer gezielten Therapie mit Tyrosinkinaseinhibitoren zugeführt werden konnten.

Bei den meisten myeloiden Neoplasien stellt die Eosinophilie einen prognostischen Faktor bezüglich der Überlebensdauer dar [15]. Zu erwähnen ist, dass es Koexistenzen verschiedener myeloider Neoplasien, zum Beispiel systemische Mastozytose und CEL, geben kann.

\section{Klassifikation der Hypereosinophiliesyndrome \\ $\nabla$}

Eine multidisziplinäre Arbeitsgruppe hat anlässlich der Working Conference on Eosinophil Disorders and Syndromes 2011 in Wien eine Aktualisierung und Anpassung der Klassifikation der Hypereosinophiliesyndrome vorgeschlagen, in die die gegenwärtig gültigen WHO-Definitionen neoplastischer Erkrankungen eingehen $[15,16]$. Das Klassifikationsschema basiert analog zu dem der Hypereosinophilie auf der zugrunde liegenden Ätiologie und umfasst die idiopathischen, die primär klonalen oder neoplastischen und die sekundären reaktiven Hypereosinophiliesyndrome $[15,16]$. Als Sonderform wurde die lymphozytäre Variante hervorgehoben, die Merkmale der reaktiven Hypereosinophilie 


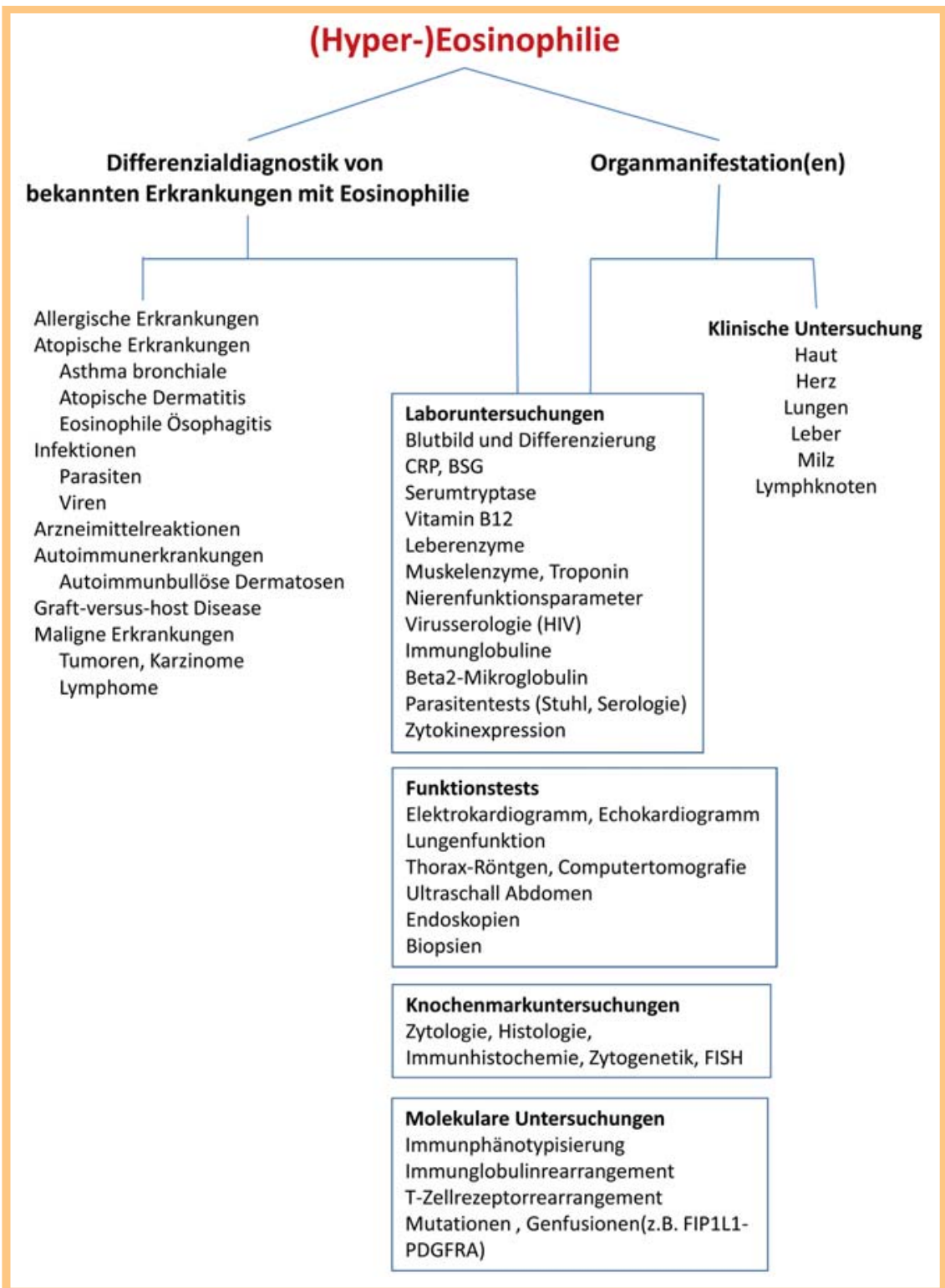

Abb. 2 Abklärungen bei (Hyper-)Eosinophilie $[2,27]$.

besitzt und durch das Auftreten aberranter T-Zellen, gelegentlich monoklonales T-Zellrezeptor-Rearrangement und meist indolenten Verlauf gekennzeichnet ist [15].

Da der Begriff Hypereosinophilie mit unbekannter Signifikanz $\left(\mathrm{HE}_{\mathrm{us}}\right)$ eine asymptomatische Hypereosinophilie ohne Zeichen einer Organschädigung beschreibt, sollte bei Auftreten von Symptomen, die durch die Hypereosinophilie bedingt sind, der Begriff des idiopathischen Hypereosinophiliesyndroms benutzt werden [16]. Die familiäre Hypereosinophilie und Hypereosinophilie mit unbekannter Signifikanz stellen somit provisorische Diagnosen dar, ein möglicher Übergang in ein Hypereosinophiliesyndrom muss engmaschig überwacht werden [16].

Verschiedene Syndrome und auf einzelne Organe beschränkte Erkrankungen sind mit einer Hypereosinophilie assoziiert, wobei die Pathogenese und damit Rolle der Eosinophilen meist unklar bleibt. Zu den Beispielen zählen das episodische Angioödem mit Eosinophilie (Gleich-Syndrom), das häufig mit dem Nachweis klonaler T-Zellen als mögliche Sonderform der lymphozytären Variante des Hypereosinophiliesyndroms einhergeht, das Churg-Strauss und das Eosinophile-Myalgie-Syndrom im Zusammenhang mit der Einnahme von L-Tryptophan sowie eosinophile
Pneumonie, eosinophiles intrinsisches Asthma, eosinophile Sinusitis, eosinophile Gastroenteritis und eosinonophile Dermatitis $[2,16,25]$.

\section{Diagnostischer Algorithmus}

Häufig stellen sich Patienten mit Hypereosinophilie wegen des unerträglichen Juckreizes vor. Die Abklärung einer Blut- und Gewebseosinophilie beginnt mit der Suche nach den häufigsten Ursachen einer (Hyper-)Eosinophilie, wobei nach allergischen Erkrankungen, arzneimittelbedingten Reaktionen und Infektionen, hierbei v.a. parasitärer Herkunft, sowie Autoimmunerkrankungen gefahndet werden muss ( $\bullet$ Abb.2) [18].

Die initialen Abklärungen umfassen eine ausführliche Anamnese einschließlich Medikamentenanamnese. Eine Stuhlanalyse oder Serologie zum Ausschluss einer Parasiteninfektion sollte bei anamnestischen Risikokonstellationen erfolgen.

Ist kein zeitlicher Zusammenhang mit einer neu begonnenen Medikation vorhanden, so muss mittels Anamnese, klinischer und laboranalytischer Untersuchungen eine Atopie (totales IgE, spezifisches IgE auf Umweltallergene als Hinweis dafür), Allergien oder Autoimmunerkrankung ausgeschlossen werden [15]. 
Bei Verdacht auf eine Vaskulitis sind die Serum-ANCA zu bestimmen. Frühe Hinweise für eine hämatologische Neoplasie kann man durch Routineanalysen wie das Blutbild und Differenzialblutbild, Leber- und Nierenwerte, Entzündungsparameter, Tryptase und Vitamin B12 gewinnen [15]. Sollten neben der Eosinophilie andere Auffälligkeiten des Blutbildes oder auch eine Splenomegalie vorliegen, muss eine Knochenmarkanalyse mit Zytologie, Histologie, Immunhistochemie und Zytogenetik veranlasst werden [14-16]. Bei Verdacht auf eine lymphozytäre Variante sollte eine Lymphozytenphänotypisierung mittels Flowzytometrie und Untersuchungen auf T-Zellrezeptor-Rearrangements erfolgen [20].

Parallel zur Suche nach der möglichen Ursache der Hypereosinophiliesyndrome sollten gleichzeitig auch Abklärungen bezüglich der Endorganschädigung(en) erfolgen. Dies umfasst eine genaue Untersuchung der Haut, eine kardiologische Beurteilung mittels EKG und Echokardiografie, eine Lungenfunktionsprüfung, Röntgenbild des Thorax sowie eine Sonografie des Abdomens. Weitere Abklärungen zur Suche einer gastrointestinalen Beteiligung sollten bei anamnestischen Hinweisen erfolgen [14,15]. Die Bestimmung von eosinophilopoetischen Zytokinen wie IL-3, IL-5 und GM-CSF im Serum kann für die Diagnose einer reaktiven Eosinophilie hilfreich sein, wird jedoch nicht routinemäßig durchgeführt. [14] Bei In-vitro-Stimulationen der T-Zellen sollte beachtet werden, dass die gemessenen Zytokine nicht unbedingt die In-vivo-Verhältnisse widerspiegeln $[18,26]$.

\section{Therapieansätze}

Da es sich bei den Hypereosinophiliesyndromen um eine sehr heterogene Gruppe meist seltener Erkrankungen handelt, ist eine genaue Charakterisierung der zugrunde liegenden Pathomechanismen notwendig, um eine möglichst spezifische Therapie anstreben zu können [27]. Als Beispiel ist der Nachweis von FIP1L1-PDGFRA mittels PCR oder FISH zu nennen, da FIP1L1PDGFRA-positive myelodysplastische Neoplasien sehr gut auf Tyrosinkinasehemmer wie Imatinib ansprechen, während bei FIP1L1-PDGFRA-negativen Formen einer Hypereosinophilie eher eine antientzündliche Behandlung indiziert ist. Bis auf eine retrospektive Multizenterstudie [28] existieren keine größeren Studien zur Behandlung der Hypereosinophiliesyndrome, meist liegen kleinere Serien oder Kasuistiken vor.

Bei rascher Progredienz und/oder lebensgefährlichen Organschädigungen sollte selbst bei fehlendem Nachweis einer spezifischen Ätiologie der Bluteosinophilie möglichst schnell eine Therapie mit hochdosierten intravenösen Kortikosteroiden eingeleitet werden (mindestens $1 \mathrm{mg} / \mathrm{kg}$ Prednisonäquivalent) [27]. Da Kortikosteroide in der Regel rasch die Eosinophilenzahlen im Blut und Gewebe senken, ist vor Therapieeinleitung eine minimale Diagnostik einschließlich Biopsien und Computertomografie zur Evaluation einer Lymphadenopathie oder Splenomegalie vorzunehmen. Bei potenzieller Exposition zu Strongyloides wird eine gleichzeitige empirische Therapie mit Ivermectin empfohlen [27]. Bei fehlendem Ansprechen auf Kortikosteroide muss ein zweites Medikament hinzugefügt werden. Hierfür gibt es keine generelle Empfehlung, die Auswahl erfolgt nach der Akuität und klinischen Manifestation, zum Beispiel Cyclophosphamid bei Verdacht auf Churg-Strauss-Syndrom, Interferon-alpha oder auch Hydroxyurea $[15,27]$.

Bei Hypereosinophilie (>1,5×109/1) aber fehlenden Symptomen oder Zeichen einer Organschädigung kann in der Regel mit einer Therapie zugewartet werden [27]. Bei Patienten mit einer Hypereosinophilie unbekannter Signifikanz $\left(\mathrm{HE}_{\mathrm{US}}\right)$ und einer familiären Hypereosinophilie $\left(\mathrm{HE}_{\mathrm{FA}}\right)$ scheinen regelmäßige Kontrollen bezüglich einer Endorganschädigung, und damit die Überprüfung der Diagnose ohne begleitende Therapie gerechtfertigt [15].

In den meisten Fällen lässt sich eine Hypereosinophilie mit Kortikosteroiden kontrollieren, eine Ausnahme sind Patienten mit myeloproliferativen Formen [15]. Die Bestimmung der genauen zytomorphologischen Eigenschaften bei myeloproliferativen Prozessen ist unabdingbar. Bei CEL ist insbesondere die Bestimmung von Fusionsproteinen wie dem FIP1L1-PDGFRA von klinischer Relevanz, da diese Patienten in der Regel ein gutes Ansprechen auf eine Therapie mit Imatinib zeigen [29]. Imatinib stellt in diesem Kontext die Erstlinientherapie dar, mit der sich auch Komplikationen wie Endokardfibrose und Thromboembolien verhindern lassen [29]. Nur wenige Patienten sprechen nicht auf Imatinib an, besitzen eine primäre oder sekundäre Resistenz [15, 30,31]. Bei Nichtansprechen auf Tyrosinaseinhibitoren muss eine Knochenmarktransplantation in Erwägung gezogen werden. Bei Hypereosinophilie aufgrund anderer Mutation wie JAK2, FGFR1 oder zytogenetischer Abnormalitäten können Interferon-alpha und Hydroxyurea eingsetzt werden. Da bei einigen Patienten mit FIP1L1-PFGFRA-negativem Hypereosinophiliesyndrom ein Ansprechen auf Imatinib beobachtet wurde, kann dieses bei Nichtansprechen auf Kortikosteroide durchaus probiert werden. Ist nur ein Organ durch eine Hypereosinophilie betroffen, so sollte die Therapie, wenn möglich, mit topischen, ansonsten mit systemischen Kortikosteroiden behandelt werden. Bei Patienten mit einem Churg-Strauss-Syndrom hat der frühzeitige Einsatz von Cyclophosphamid zusammen mit Kortikosteroiden eine Reduktion der Mortalität und Morbidität bewirkt [27]. Desweiteren wurden erfolgreiche Behandlungen mit Methotrexat, Hydroxyurea und Interferon-alpha berichtet. Mepolizumab, ein Anti-IL5-Antikörper, erwies sich als sicher und gut wirksam in der Behandlung von Hypereosinophiliesyndromen und Churg-StraussSyndrom [32-34].

Die Behandlung der lymphozytären Formen der Hypereosinophiliesyndrome mit klonalen oder aberranten T-Zellpopulationen erfordert häufig hohe Dosen von Kortikosteroiden, sodass Interferon-alpha eine steroidsparende Alternative darstellt [27]. Der Effekt von Ciclosporin ist meist nicht ausreichend [27]. Bei reaktiver Hypereosinophile muss die Grunderkrankung behandelt werden, was zu einer Reduktion bzw. Verschwinden eosinophilopoetischer Zytokine und damit einer Abnahme der Eosinophilenzahlen im Blut und/oder Gewebe führt.

\section{Ausblick \\ $\nabla$}

So wie wir lernen, die unterschiedlichen Ursachen, die zu einer Hypereosinophilie führen, und die pathogenische Rolle der Eosinophilen zu verstehen, werden sich neue Therapiemöglichkeiten der Hypereosinophiliesyndrome ergeben. Die Entdeckung der FIP1L1-PDGFRA-Mutationen und die sich daraus ergebende gezielte Therapie mit Tyrosinkinaseinhibitoren waren ein erster Durchbruch. Inzwischen kennen wir eine Vielzahl von Oberflächenmolekülen, einschließlich Zytokin-, Adhäsions- und Chemokinerezeptoren, die als potenzielle Angriffspunkte einer zielgerichteten Therapie einer Eosinophilen-vermittelten Erkrankung gelten. Neue Erkenntnisse sind von Studien zum Einsatz der Anti-IL-5-Antikörper zu erwarten. 


\section{Interessenkonflikt}

$\nabla$

Die Autoren geben an, dass kein Interessenkonflikt besteht.

\section{Abstract}

\section{Hypereosinophilic Syndromes}

In recent years, the knowledge on eosinophil biology as well as their role in health and disease has dramatically increased. Differential insights in the pathogenesis, new diagnostic techniques and therapeutic substances have modified our understanding of hypereosinophilia and associated diseases and syndromes. In this review, we discuss the current classification of hypereosinophilic syndromes as well as therapeutic strategies.

\section{Literatur}

1 Chusid MJ, Dale DC, West BC et al. The hypereosinophilic syndrome: analysis of fourteen cases with review of the literature. Medicine (Baltimore) 1975; 54: 1-27

2 Simon HU, Rothenberg ME, Bochner BS et al. Refining the definition of hypereosinophilic syndrome. J Allergy Clin Immunol 2010; 126: 45 49

3 Straumann A, Simon HU. The physiological and pathophysiological roles of eosinophils in the gastrointestinal tract. Allergy 2004; 59: $15-25$

4 Simon D, Braathen LR, Simon HU. Eosinophils and atopic dermatitis. Allergy 2004; 59: $561-570$

5 Blanchard C, Rothenberg ME. Biology of the eosinophil. Adv Immunol 2009; 101: $81-121$

6 Gleich GJ. Mechanisms of eosinophil-associated inflammation. J Allergy Clin Immunol 2000; 105: 651-663

7 Yousefi S, Gold JA, Andina N et al. Catapult-like release of mitochondrial DNA by eosinophils contributes to antibacterial defense. Nat Med 2008; 14: 949-953

8 Morshed M, Yousefi S, Stockle C et al. Thymic stromal lymphopoietin stimulates the formation of eosinophil extracellular traps. Allergy 2012; 67: 1127-1137

9 Dworski R, Simon HU, Hoskins A et al. Eosinophil and neutrophil extracellular DNA traps in human allergic asthmatic airways. J Allergy Clin Immunol 2011; 127: 1260-1266

10 Kerstan A, Simon HU, Yousefi S et al. Extensive accumulation of eosinophil extracellular traps in bullous delayed-pressure urticaria: a pathophysiological link? Br J Dermatol 2012; 166: 1151-1152

11 Simon D, Hoesli $S$, Roth $N$ et al. Eosinophil extracellular DNA traps in skin diseases. J Allergy Clin Immunol 2011; 127: 194-199

12 Simon D, Simon HU, Yousefi S. Extracellular DNA traps in allergic, infectious, and autoimmune diseases. Allergy 2013; 68: 409-416

13 Roth N, Stadler S, Lemann M et al. Distinct eosinophil cytokine expression patterns in skin diseases - the possible existence of functionally different eosinophil subpopulations. Allergy 2011; 66: 1477-1486

14 Valent $P$. Pathogenesis, classification, and therapy of eosinophilia and eosinophil disorders. Blood Rev 2009; 23: 157-165
15 Valent P, Gleich GJ, Reiter A et al. Pathogenesis and classification of eosinophil disorders: a review of recent developments in the field. Expert Rev Hematol 2012; 5: 157-176

16 Valent P, Klion AD, Horny HP et al. Contemporary consensus proposal on criteria and classification of eosinophilic disorders and related syndromes. J Allergy Clin Immunol 2012; 130: 607-612

17 Wilkins HJ, Crane MM, Copeland $K$ et al. Hypereosinophilic syndrome: an update. Am J Hematol 2005; 80: 148-157

18 Simon D, Simon HU. Eosinophilic disorders. J Allergy Clin Immunol 2007; 119: 1291 - 1300

19 Fridlender ZG, Simon HU, Shalit M. Metastatic carcinoma presenting with concomitant eosinophilia and thromboembolism. Am J Med Sci 2003; 326: $98-101$

20 Simon HU, Plotz SG, Dummer R et al. Abnormal clones of T cells producing interleukin-5 in idiopathic eosinophilia. N Engl J Med 1999; 341: $1112-1120$

21 Simon HU, Plotz SG, Simon D et al. Clinical and immunological features of patients with interleukin-5-producing $\mathrm{T}$ cell clones and eosinophilia. Int Arch Allergy Immunol 2001; 124: 242 - 245

22 Lammel V, Stoeckle C, Padberg B et al. Hypereosinophilia driven by GMCSF in large-cell carcinoma of the lung. Lung Cancer 2012; 76: 493495

23 Tefferi A, Vardiman JW. Classification and diagnosis of myeloproliferative neoplasms: the 2008 World Health Organization criteria and point-of-care diagnostic algorithms. Leukemia 2008; 22: 14-22

24 Wadleigh $M$, Tefferi A. Classification and diagnosis of myeloproliferative neoplasms according to the 2008 World Health Organization criteria. Int J Hematol 2010; 91: 174-179

25 Simon D, Wardlaw A, Rothenberg ME. Organ-specific eosinophilic disorders of the skin, lung, and gastrointestinal tract. J Allergy Clin Immunol 2010; 126: 3-13

26 Stoeckle C, Simon HU. CD8(+) T cells producing IL-3 and IL-5 in non-IgEmediated eosinophilic diseases. Allergy 2013; 68: 1622-1625

27 Simon HU, Klion A. Therapeutic approaches to patients with hypereosinophilic syndromes. Semin Hematol 2012; 49: 160-170

28 Ogbogu PU, Bochner BS, Butterfield JH et al. Hypereosinophilic syndrome: a multicenter, retrospective analysis of clinical characteristics and response to therapy. J Allergy Clin Immunol 2009; 124: 13191325

29 Klion $A D$, Noel P, Akin $C$ et al. Elevated serum tryptase levels identify a subset of patients with a myeloproliferative variant of idiopathic hypereosinophilic syndrome associated with tissue fibrosis, poor prognosis, and imatinib responsiveness. Blood 2003; 101: 4660 - 4666

30 Salemi S, Yousefi S, Simon D et al. A novel FIP1L1-PDGFRA mutant destabilizing the inactive conformation of the kinase domain in chronic eosinophilic leukemia/hypereosinophilic syndrome. Allergy 2009; 64: 913-918

31 Simon D, Salemi S, Yousefi $S$ et al. Primary resistance to imatinib in Fip1-like 1-platelet-derived growth factor receptor alpha-positive eosinophilic leukemia. J Allergy Clin Immunol 2008; 121: 1054- 1056

$32 \mathrm{Kim}$ S, Marigowda G, Oren E et al. Mepolizumab as a steroid-sparing treatment option in patients with Churg-Strauss syndrome. J Allergy Clin Immunol 2010; 125: 1336 - 1343

33 Plotz SG, Simon HU, Darsow U et al. Use of an anti-interleukin-5 antibody in the hypereosinophilic syndrome with eosinophilic dermatitis. N Engl J Med 2003; 349: 2334-2339

34 Rothenberg ME, Klion AD, Roufosse FE et al. Treatment of patients with the hypereosinophilic syndrome with mepolizumab. N Engl J Med 2008; 358: $1215-1228$ 